\title{
Effect of external pressure on the normal and superconducting properties of high- $T_{c}$ cuprates
}

\author{
K. A. Sidorov ${ }^{1,2}$, V. A. Gavrichkov ${ }^{1,2}$, S. V. Nikolaev ${ }^{*}$ 1, 2, Z. V. Pchelkina ${ }^{3}$, and S. G. Ovchinnikov ${ }^{1,2}$ \\ ${ }^{1}$ L.V. Kirensky Institute of Physics, SB RAS, 660036 Krasnoyarsk, Russia \\ ${ }^{2}$ Siberian Federal University, 660041 Krasnoyarsk, Russia \\ ${ }^{3}$ Institute of Metal Physics, UB RAS, 620219 Ekaterinburg, Russia
}

Received XXXX, revised XXXX, accepted XXXX

Published online XXXX

Key words: cuprates, electronic structure, superconducting properties, strongly correlated electrons, Fermi surface, Lifshitz transitions.

* Corresponding author: e-mail svinikolaev@sfu-kras.ru, Phone: +7-391-2062117, Fax: +7-391-2912967

The pressure effects on the normal state electronic structure, the superexchange interaction, and the critical temperature of d-type superconductivity mediated by magnetic pairing have been studied within the multielectron hybrid scheme LDA+GTB that takes into account electron correlations in $\mathrm{CuO}_{2}$ planes. We have found the changes of the multiband $p-d$ model parameters at $3 \%$ compression of different symmetry: a) hydrostatic, b) along the $c$-axis, c) in $a-b$ plane. We have studied the changes of the Fermi surface under external pressure for different hole doping concentration $x$. In general this effect is too small except two critical concentrations $x_{c 1} \approx$ 0.15 and $x_{c 2}=0.24$ where the Lifshitz transitions occur with the change of the Fermi surface topology. In the vicinity of the critical concentration we have found the giant change of the Fermi surface area up to $100 \%$ related to the pressure induced Lifshitz transition. The effects of pressure on the antiferromagnetic coupling $J$ and the mean-field value of $T_{c}$ are obtained in a good agreement to experimental data.

Copyright line will be provided by the publisher

1 Introduction In spite of a remarkable progress in physics of high- $T_{c}$ superconducting cuprates our understanding of the unusual normal state in a wide doping and temperature range as well as the origin of superconducting pairing is far from being complete. In this situation experimental study under external pressure $P$ is exceptionally valuable as an in situ way to probe the electronic struture and the temperature $T_{c}$ response [1]. The maximal value of $T_{c}$ for cuprates has been achieved in the optimally doped three-layer compound $\mathrm{HgBa}_{2} \mathrm{Ca}_{2} \mathrm{Cu}_{3} \mathrm{O}_{8+y}(\mathrm{Hg}-1223)$ with $T_{c} \approx 134 K$ at ambient pressure [2], reaching a value near $160 \mathrm{~K}$ at hydrostatic pressure $30 \mathrm{GPa}$ [3]. A combination of the hydrostatic and uniaxial pressure results in a separation of different structural effects on the electronic structure parameters and the hydrostatic $d T_{c} / d P$ and uniaxial pressure derivatives $d T_{c} / d P_{i}(i=a, b, c)$ [4-6].

Empirically, several correlations of $T_{c}$ and structural parameter changes under pressure have been revealed $[1,7$, 8]. Two key structural quantities have been suggested: the
$\mathrm{CuO}_{4}$ placket area (or the bond length $l$ between copper and in-plane oxygen), and the $\mathrm{Cu}$-apical oxygen distance $h_{o}$. The role of the apical oxygen on the electronic properties has been addressed in several theoretical works [9-13] as the effect of interlayer coupling in the phonon-mediated BCS-like theory [14].

Recently, the time-frequency resolved spectroscopy [15] has revealed a dominant role of the non-retarding electronic mechanism of pairing. The antiferromagnetic nearest neighbor exchange interaction $J$ is one of the candidates for the electronic pairing. The experimental studies [16-18] have found $d J / d P>0$ under hydrostatic pressure. However we didnt find any publications concerning the uniaxial pressure effect on $J$. The normal state electronic properties as the electronic band structure and the Fermi surface under external pressure are less studied. It is clear that a consistent theoretical description and new predictions of the pressure effects on the properties of both normal and superconducting phases may results in a more 
profound understanding of the unsolved problems of the high $T_{c}$ cuprates.

In this paper we have realized this program within the hybrid multielectron approach LDA+GTB [19,20], where LDA is the conventional local density approximation to the density functional theory, and GTB is the generalized tight binding method proposed for strongly correlated electronic systems [21,22]. The GTB method is a version of the cluster perturbation theory with all local interactions inside the $\mathrm{CuO}_{4}\left(\mathrm{CuO}_{6}\right)$ unit cell treated by exact diagonalization and the intercell hopping $t$ by the perturbation theory over $t / E_{c t}$ where $E_{c t}$ is the charge transfer gap of the parent undoped insulator $\left(\mathrm{La}_{2} \mathrm{CuO}\right.$ for $\mathrm{La}_{2-x} \mathrm{Sr}_{x} \mathrm{CuO}_{4}$ ). Within the LDA+GTB approach at ambient pressure we have calculated the exchange interaction $J=0.14 e \mathrm{~V}$ for $\mathrm{La}_{2} \mathrm{CuO}_{4}$ [23], and have shown that with doping there are two Lifshits transitions with the change of the Fermi surface. At $x_{c 1}=0.15$ four small hole pockets centered around $(\pi / 2, \pi / 2)$ transformed into two hole pockets around $(\pi, \pi)$. At $x_{c 2}=0.24$ the small pocket disappears and at $x>0.24$ only one large hole Fermi surface remains [24,25].

Under external hydrostatic and uniaxial pressure we have calculated the changes of the tight-binding parameters for the 5-band $p$ - $d$ model within the LDA approach in Sec. 2. In Sec. 3 we give a brief outlook of the LDA+GTB method for the electronic structure in the spin liquid magnetic background with a strong short-range antiferromagnetic order. The changes of the Fermi surface area under the external pressure for different doping value are discussed in Sec. 4. The pressure effect on the exchange interaction is given in Sec. 5. Sec. 6 contains the effect of pressure on $T_{c}$ that results from both the changers of the normal state electronic structure and the coupling constant $J$. The discussion of results is given in Sec. 7 .

2 Deformation dependence of tight-binding parameters for the 5 -band $p$ - $d$ model within the LDA approach We model the electronic system of a $\mathrm{CuO}$ layer in cuprates within the multiband $p$ - $d$ model [26] that includes two $C u$ orbitals: $d_{x^{2}-y^{2}}$ (noted as $d_{x^{2}}$ below) and $d_{3 z^{2}-r^{2}}\left(d_{z^{2}}\right)$, and three orbitals of oxygen: $p_{x}, p_{y}$ for inplane oxygen and $p_{z}$ for apical oxygen. The Hamiltonian of this model includes the local single-hole energies at different orbitals, the hopping matrix elements, and the intraatomic Coulomb interactions on $C u$ and $O$, it has been discussed in many papers (see, f.e. $[19,20,22]$ ) and we do not repeat it here. The tight-binding parameters of the Hamiltonian at ambient pressure has been calculated for $\mathrm{La}_{2-x} \mathrm{Sr}_{x} \mathrm{CuO}_{4}$ by construction of the Wannier functions of the same symmetry starting from the LDA approach [19]. Here we use the same approach to find the changes of the tight-binding parameters under external pressure.

We simulate the effect of applied pressure as follows (see Fig. 1): (a)

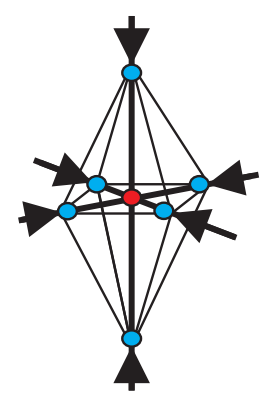

$\mathrm{V}(\mathrm{P})=0.97 \mathrm{~V}_{0}$ (b)

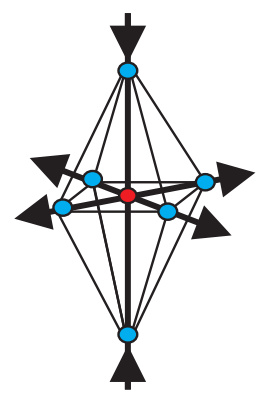

$\mathrm{V}\left(\mathrm{P}_{\mathrm{c}}\right)=\mathrm{V}_{0}$ (c)

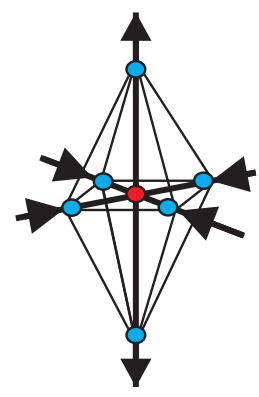

$\mathrm{V}\left(\mathrm{P}_{\mathrm{ab}}\right)=\mathrm{V}_{0}$
Figure 1 Three ways of microscopic simulation of the $\mathrm{CuO}$ octahedron compression induced by (a) hydrostatic, (b) uniaxial and (c) in-plane applied pressure. The expansion of the $\mathrm{CuO}_{4}$ squares in the (b) case and elongation of $c$ parameter in the (c) case were derived from an empirical condition of constant unit cell volume.

- as known up to $\sim 15 G P a$ the ratio $c / a(b)$ remains constant showing microscopic picture isotropic [27]. Thus the hydrostatic pressure $P$ effect is simulated by the $3 \%$ deformation of the unit cell volume $V(P)$ and $\sim 1 \%$ deformation in the unit cell linear size $d(P)=$ $\sqrt[3]{0.97} d$, where $d=a, b, c$ is a lattice parameter;

- uniaxial pressure along the $c$-axis $P_{c}$ effect is simulated by the $3 \%$ reduction in the structural $c$-parameter at the constant unit cell volume $V\left(P_{c}\right)$;

- in-plane $P_{a b}$ pressure effect is simulated by the $\sim 1 \%$ reduction in the structural $a$ and $b$ parameters $a\left(P_{a b}\right)$ and $b\left(P_{a b}\right)$ at the constant unit cell volume $V\left(P_{a b}\right)$.

In the first and last cases, we have selected the equivalent magnitudes of in-plane deformation (with opposite strains along $c$-axis) in order to be able to highlight the superexchange $J(P)$ dependence on the ratio $c / a(b)$. Despite the fact that the table shows the same vectors, a system of the connecting vectors varies slightly with increasing pressure. Unfortunately, we cant study the uniaxial pressure effects along $\mathrm{a}$ and $\mathrm{b}$ axis in this approach, because it changes the Wannier functions symmetry.

The last line in Table 1 containes the value of the superexchange interaction that will be discussed below in Sec. 5.

3 LDA+GTB method for the electronic structure in the spin liquid magnetic background with strong short-range antiferromagnetic order The cell approach of the generalized tight binding method [22, 28 ] is used to take into account strong electron correlations in the unit cell explicitly. A crystal lattice is divided into unit cells, so that the Hamiltonian is presented by $H=H_{0}+H_{1}$, where the component $H_{0}$ is the sum of intracell terms and the component $H_{1}$ takes into account 
Table 1 Structural parameters $a, b, c$, single electron energies, hopping parameters, $J(P)$ for orthorhombic $L a 214$ (all values except the connecting vectors in $e V$ ). Here $x^{2}, z^{2}, p_{x}, p_{y}, p_{z}$ denote $C u-d_{x^{2}-y^{2}}, C u-d_{3 z^{2}-r^{2}}, O-p_{x}, O-p_{y}, O-p_{z}$ orbital respectively.

\begin{tabular}{llllll}
\hline Parameters & Connecting vectors & $\begin{array}{l}3 \% \text { compression } \\
\text { along } c \text { axis }\end{array}$ & $\begin{array}{l}\text { Undeformed } \\
\text { terial }\end{array}$ & $\begin{array}{l}3 \% \text { hydrostatic } \\
\text { compression }\end{array}$ & $\begin{array}{l}\text { In-plane compres- } \\
\text { sion }\end{array}$ \\
\hline $\mathrm{a}$ & & 5.416 & 5.335 & 5.281 & 5.281 \\
$\mathrm{~b}$ & & 5.498 & 5.415 & 5.360 & 5.360 \\
$\mathrm{c}$ & & 12.724 & 13.117 & 12.985 & 13.386 \\
$\epsilon_{x^{2}}$ & & -2.031 & -1.849 & -2.174 & -2.456 \\
$\epsilon_{x^{2}}-\epsilon_{z^{2}}$ & & 0.119 & 0.225 & 0.191 & 0.215 \\
$\epsilon_{x^{2}}-\epsilon_{p_{x}}$ & & 0.983 & 0.957 & 0.965 & 0.952 \\
$\epsilon_{x^{2}}-\epsilon_{p_{y}}$ & & 0.983 & 0.957 & 0.965 & 0.952 \\
$\epsilon_{x^{2}}-\epsilon_{p_{z}}$ & & -0.503 & -0.173 & -0.659 & -0.614 \\
$t\left(x^{2}, x^{2}\right)$ & $(-0.493,-0.5)$ & -0.173 & -0.188 & -0.187 & -0.188 \\
$t\left(z^{2}, z^{2}\right)$ & $(-0.493,-0.5)$ & 0.050 & 0.054 & 0.054 & 0.052 \\
$t\left(x^{2}, p_{x}\right)$ & $(0.246,0.25,-0.02)$ & 1.302 & 1.355 & 1.424 & 1.422 \\
$t\left(z^{2}, p_{x}\right)$ & $(0.246,0.25,-0.02)$ & -0.547 & -0.556 & -0.571 & -0.548 \\
$t\left(z^{2}, p_{z}\right)$ & $(0,0.5,0.041)$ & 0.851 & 0.773 & 0.811 & 0.748 \\
$t\left(p_{x}, p_{y}\right)$ & $(0.493,0.0)$ & -0.854 & -0.858 & -0.882 & -0.889 \\
$t\left(p_{x}, p_{z}\right)$ & $(-0.246,-0.21,0.465)$ & -0.447 & -0.391 & -0.409 & -0.370 \\
$J(\Delta J \%)$ & & $0.140(-5.7 \%)$ & 0.149 & $0.160(+7.4 \%)$ & $0.162(+8.2 \%)$ \\
\hline
\end{tabular}

the intercell hopping and interactions. The component $H_{0}$ is exactly diagonalized. The exact multielectron cell states $|n, \Theta\rangle$ with energies $E_{n \Theta}$ are determined, where index $n$ numerates the eigenstates within three subsectors of the Gilbert space with hole numbers $n_{h}=0$ (hole vacuum $d^{10} p^{6}$ with spin $\left.S=0\right), n_{h}=1\left(\operatorname{mixed} d^{9} p^{6}\right.$ and $d^{10} p^{5}$ configurations), and $n_{h}=2\left(\operatorname{mixed} d^{9} p^{5}, d^{10} p^{4}, d^{8} p^{6}\right.$ configurations), spin index $\Theta=\sigma, S, M$ for the single hole doublet with spin projection $\sigma= \pm 1$, two hole singlet $S=0$ and triplet states $M=0,+1,-1$. Then these states are used to construct the Hubbard operators of the unit cell $\mathbf{R}_{\mathbf{f}}: X_{f}^{n \Theta, n^{\prime} \Theta^{\prime}}=|n \Theta\rangle\left\langle n^{\prime} \Theta^{\prime}\right|$. Thereafter, the component $H_{1}$ is exactly written in the $X$-operator representation and intercell interactions are included in terms of the perturbation theory. The cluster perturbation theory for the electronic structure, the superexchange interaction and the magnetic mechanism of pairing at ambient pressure for the undeformed $\mathrm{CuO}$ layer are described in our previous reviews $[20,29]$. Here we have presented in brief the main ideas and results of the LDA+GTB approach that is necessary to understand the forthcoming results of Sec. 4. The electron (and hole) in this approach is described as a linear combination of different fermionic quasipartices, each of them is the excitations between an initial and a final multielectron terms $|n \Theta\rangle$ with change of the electronic number \pm 1 . For example, excitations from $|1 \sigma\rangle$ to $|0\rangle$ configurations is the electron addition quasiparticle forming the conductivity band. Various Fermi-type excitations from $|1 \sigma\rangle$ to $|2, S\rangle$ and $|2, M\rangle$ corresponds to the electron removal quasiparticles forming subbands of the valence band. All such quasiparticles are fermionic and have the electric charge 1 and spin $1 / 2$, nevertheless it is convenient to call there subbands as singlet and triplet bands depending on the type of the final state. Thus, in more simplified three band $p$ - $d$ model the term singlet band is often used for the fermionic band forming with participation of the Zhang-Rice singlet.

For undoped $\mathrm{La}_{2} \mathrm{CuO}_{4}$ the LDA+GTB approach results in the charge transfer insulator with the gap $E_{c t}=1.7 \mathrm{eV}$ [20]. To study the electronic structure at small excitation energies and the Fermi surface one may simplify the problem by constructing the effective low energy Hamiltonian. The low-energy Hamiltonian for $\mathrm{La}_{2 x} \mathrm{Sr}_{x} \mathrm{CuO}_{4}$ (LSCO) is the $t-t^{\prime}-t^{\prime \prime}-J^{*}$-model obtained via exclusion of the interband (through the charge-transfer gap) excitations. Here $J^{*}$ means that besides the Heisenberg exchange term a three-site correlated hopping $H_{3}$ is also included $H_{t-J^{*}}=H_{t-J}+H_{3}$. In the wide range of hole doping beyond the long range antifferomagnetic order at $x<0.03 \mathrm{LSCO}$ is characterized by a short range antiferromagnetic order that had been described as an isotropic spin liquid with zero mean value of every projection of local spin $\left\langle S^{x}\right\rangle=\left\langle S^{y}\right\rangle=\left\langle S^{z}\right\rangle=0$ and nonzero spin correlation functions $C_{i j}$ at different sited $R_{i}, R_{j}, C_{i j}=\left\langle S_{i}^{r} S_{j}^{r}\right\rangle$ is the same for all spin projections $r=x, y, z$. These correlation functions determines the electronic self energy $\Sigma(\mathbf{k})$ of the electronic Green function $G_{k \sigma}(E)$ in the non crossing diagram approximation [24]

$$
G_{\mathbf{k} \sigma}(E)=\frac{(1+x) / 2}{E-\epsilon+\mu-\frac{1+x}{2} t(\mathbf{k})-\frac{1-x^{2}}{4} \frac{\tilde{t}^{2}}{U}-\Sigma(\mathbf{k})} .
$$


(a)

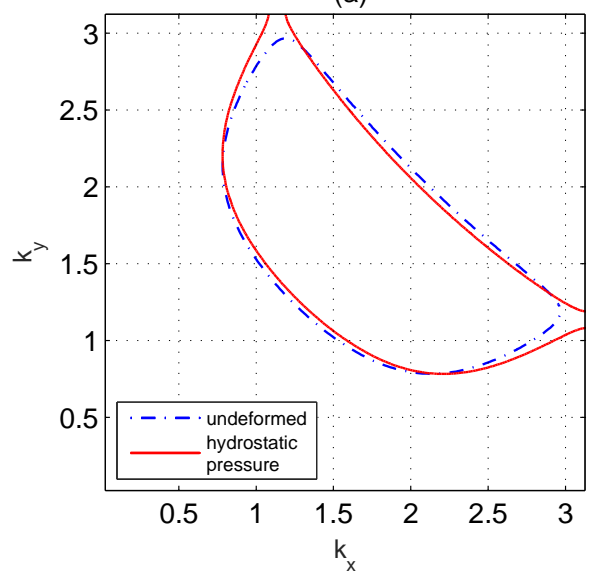

(b)

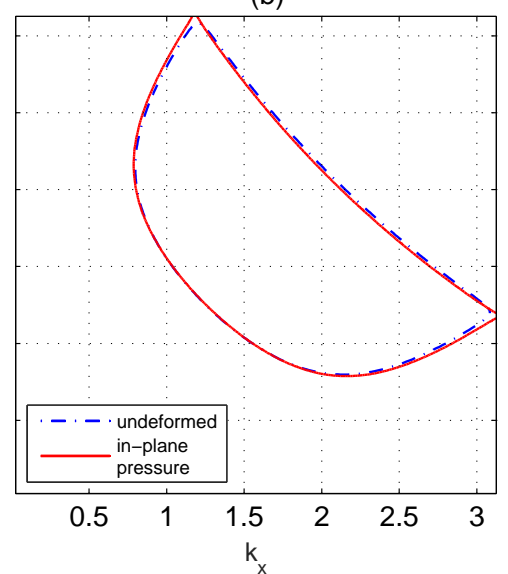

(c)

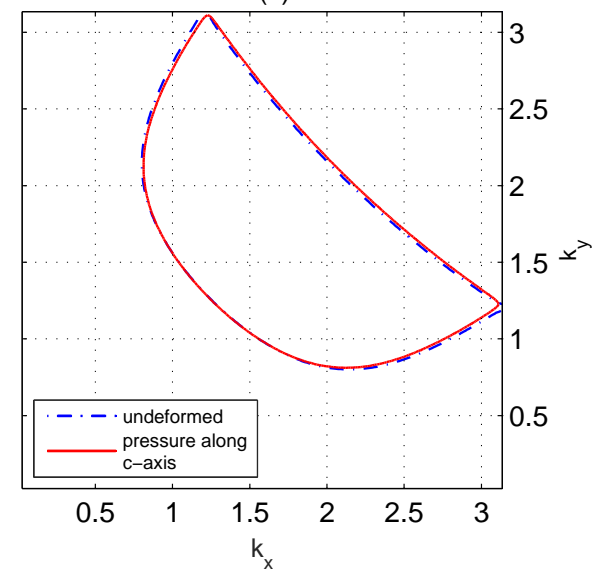

Figure 2 (a) The splitting of the Fermi surface for hydrostatic pressure at $x=0.145$. The relative change in the area $\delta_{\text {small }}=-7.1 \%$ for a small pocket and $\delta_{\text {large }}=92.9 \%$ for a large pocket. (b) The splitting of the Fermi surface for the pressure in the $a$ - $b$ plane at $x=0.15\left(\delta_{\text {small }}=-14.0 \%, \delta_{\text {large }}=85.9 \%\right)$. (c) The splitting of the Fermi surface for the pressure along $c$-axis at $x=0.151$ $\left(\delta_{\text {small }}=17.1 \%, \delta_{\text {large }}=-82.9 \%\right)$. Here $\delta_{\text {small(large })}=\left(S_{\text {small(large })}^{(\text {presure }}-S^{(\text {undeformed })}\right) / S^{(\text {undeformed })} \cdot 100 \%$ for (a) and (b) $)$ and $\delta_{\text {small(large })}=\left(S^{(\text {pressure })}-S_{\text {small(large })}^{(\text {undeformed })}\right) / S^{(\text {pressure })} \cdot 100 \%$ for (c).

Here $\epsilon$ is the single $d_{x^{2}}$-hole energy, $\mu$ is the chemical potential, $t(\mathbf{k})$ is the Fourier transform of the intercell hopping with $t, t^{\prime}, t^{\prime \prime}$ hopping parameters, $\tilde{t}(\mathbf{k})$ is similar function with the interband hopping parameters $\tilde{t}, \tilde{t}^{\prime}, \tilde{t}^{\prime \prime}$ (hopping from site $\mathbf{R}_{i}$ to site $\mathbf{R}_{j}$ and from upper Hubbard band to low Hubbard throw the gap $U$ ). The self energy $\Sigma(\mathbf{k})$ is given by

$$
\Sigma(\mathbf{k})=\frac{1}{N} \frac{2}{1+x} \sum_{\mathbf{q}}\left\{Y_{1} K(\mathbf{q})-\frac{3}{2} Y_{2} C(\mathbf{q})\right\},
$$

where

$$
\begin{aligned}
Y_{1}= & t(\mathbf{q})-\frac{1-x}{2} J(\mathbf{k}-\mathbf{q})-x \frac{\tilde{t}^{2}(\mathbf{q})}{U}-(1+x) \frac{\tilde{t}(\mathbf{k}) \tilde{t}(\mathbf{q})}{U}, \\
Y_{2}= & t(\mathbf{k}-\mathbf{q})-\frac{1-x}{2}\left(J(\mathbf{q})-\frac{\tilde{t}(\mathbf{k}-\mathbf{q})}{U}\right) \\
& -(1+x) \frac{\tilde{t}(\mathbf{k}) \tilde{t}(\mathbf{k}-\mathbf{q})}{U},
\end{aligned}
$$

In these expressions $C(\mathbf{q})$ is the Fourier transform of the spin correlation function $C_{i j}$. The function $K(\mathbf{q})$ is the kinematic correlation function straightforwardly expressed via Green function (1). The spin correlation function $C(\mathbf{q})$ had been found within the isotropic spin liquid approach [30,31].

The electronic self energy, spin correlation function, and chemical potential had been found self-consistently for different doping concentration $x$ [24]. The spin correlation functions and the electronic band structure appears to be strongly doping dependent, as well as the Fermi surface. At small doping there are 4 hole pockets centered near $(\pi / 2, \pi / 2)$ in agreement with many theoretical calculations for the lightly doped antiferromagnetic insulator [3236]. Increasing doping results in the two Lifshitz transitions [37] at the critical points $x_{c 1}=0.15$ and $x_{c 2}=0.24$ $[24,25]$. For $x_{c 1}<x<x_{c 2}$ there are two hole Fermi surfaces centered at $(\pi, \pi)$, the smaller one disappears at $x_{c 2}$. Above $x_{c 2}$ the large hole Fermi surface corresponds to the conventional Fermi liquid normal state.

4 The change of the Fermi surface area under the external pressure for different doping Changing the energy parameters of the electronic structure $\mathrm{La}_{2-x} \mathrm{Sr}_{x} \mathrm{CuO}_{4}$ by external pressure results in the change of the band structure and Fermi energy, and with them the shape of the Fermi surface. The changing of the shape of the Fermi surface in turn leads to a change in the numerical value of the area of its extreme sections that may be checked by quantum oscillations experiment in strong magnetic field. As it is known, the oscillation frequency $\omega=\left(\Delta\left(\frac{1}{H}\right)\right)^{-1}$ of the magnetization in the de Haas - van Alphen effect is proportional to this area $S$ [37]:

$$
\omega=\frac{c S}{2 \pi e \hbar} .
$$

From relationship it follows that the relative change in the oscillation frequency is equal to the relative change in the area of the extremal section of the Fermi surface:

$$
\frac{\Delta \omega}{\omega}=\frac{\Delta S}{S} \text {. }
$$

We found that for $3 \%$ deformation and concentrations of the hole doping $x$ outside the region $x_{c 1}<x<x_{c 2}$ value of relative changes in the area $\delta=\frac{\Delta S}{S}<10^{-3}$. 
Such changes are too small to be revealed in the modern oscillation experiment with typical resolution in $\delta$ about 3 $4 \%$ [38]. Obviously, the maximum relative change in crosssectional area of the Fermi surface to be expected at a point where one of the surfaces (with or without pressure) splits. Fermi surfaces for these points for all three types of pressure are shown in Fig. 2. The relative changes in the area of the Fermi surfaces are shown in Table 2.

5 The pressure effect on the exchange interaction There are several approaches to study of superexchange interaction. The straightforward one is the calculation with the intermediated two-hole states which arise through hopping from copper to oxygen in perturbation theory of fourth order $[39,40]$. Another approach is a cell perturbation theory taking into account two hole excited states. Using the LDA+GTB approach and extending the cell perturbation theory to an arbitrary numbers of the excited two hole cell states, we had calculated the superexchange interaction in the $\mathrm{La}_{2} \mathrm{CuO}_{4}$ at ambient pressure [23]. The superexchange interaction appears at the second order of the cell perturbation theory with respect to the interband hopping, it is formed by the virtual excitations from occupied valence band with singlet and triplet twohole origin through the insulating gap to the conduction band and back.

We obtained that the superexchange interaction

$$
J=J_{A}-J_{B}=\sum_{n=1}^{N_{s}} \frac{t_{i j}^{0, n s}}{\Delta_{n s}}-\sum_{m=1}^{N_{T}} \frac{t_{i j}^{0, m}}{2 \Delta_{m}} .
$$

is given by a sum of the antiferromagnetic (AFM) $J_{A}$ contributions from all two-hole singlets with excitation energies $\Delta_{n s}=E_{n s}-2 E_{1 \sigma}$ and a sum of all ferromagnetic $J_{B}$ contributions from the triplet two hole states with excitation energies $\Delta_{m}=E_{m}-2 E_{1 \sigma}$. At ambient pressure the hopping matrix elements and the excitation energies had been calculated from the $a b$ initio parameters and the total superexchange interaction [23] is close to experimental value $0.146 \mathrm{eV}$ [41].

Here we have calculated the changes in the superexchange interaction under external pressure of different symmetry. The value $J(P)$ is shown in the last line of Table 1 above in Sec. 2. It is increasing by $+7.4 \%$ under $3 \%$ hydrostatic compression. At the same time, the superexchange interaction is slightly reduced by $-5.7 \%$ under the uniaxial compression along $c$-axis. The in-plane deformation results in the $J$ increase similar to the hydrostatic loading. According to the available experimental data the superexchange interaction had been increased by $+8.8 \%$ at $3 \%$ - hydrostatic compression under pressure 5.6GPa. In all cases, for hydrostatic (or in-plane) and uniaxial compressions the $J(P)$ correlates well with an area of the $\mathrm{CuO}_{4}$ squares. The magnitude of isotropic and planar compressions was chosen from the condition of equality $\mathrm{CuO}_{4}$ square areas. At the same time deformations along the $c$ axis have opposite signs, and the unit cell volume is constant.

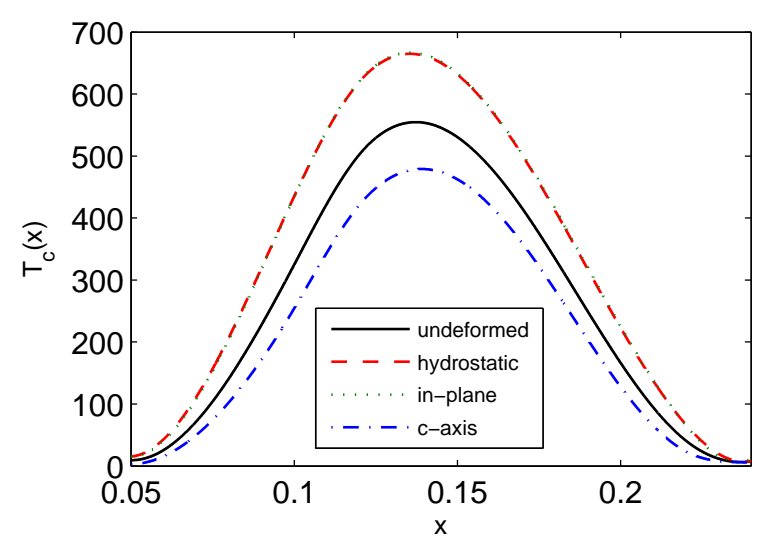

Figure 3 The superconducting temperature $T_{c}$ versus hole concentration $x$ for the absent pressure (black solid line), the hydrostatic pressure (red dashed line), the in-plane pressure (green dotted line) and the pressure along $c$-axis (blue dash-dotted line).

6 Effect of pressure on the superconducting transition temperature Within the LDA-GTB approach the AFM superexchange directly results in the $d$-type superconducting pairing similar to the Hubbard model in the strong correlation limit $[42,43]$. The mean-field theory of the $d$-type superconductivity in the limit of strong electron correlations results in the following equation for the superconducting gap $\Delta_{k}=\Delta_{0} \phi(k)$, where $\Delta_{0}$ is the gap amplitude and $\phi(k)=\left(\cos k_{x} a-\cos k_{y} a\right) / 2$ is the angular part of the order parameter

$$
\frac{1}{\lambda}=\frac{1}{N} \sum_{\mathbf{q}} \frac{4 \phi^{2}(\mathbf{q})}{\xi(\mathbf{q})-\mu} \tanh \left(\frac{\xi(\mathbf{q})-\mu}{k_{B} T}\right) .
$$

Here $k_{B}$ is the Boltzmann constant, $T$ is the temperature, $\xi(\mathbf{q})$ is the hole dispersion in the superconducting phase. The magnetic coupling constant $\lambda=J(1-x) / 2$. The external pressure effects on the renormalization of the dispersion of the normal and superconducting phases and on the magnetic coupling constant. Fig. 3 shows the dependencies $T_{c}(x)$ obtained for the ambient pressure and the external pressure of different symmetry. We can see that our values of $T_{c}$ are too large, this is an usual drawback of the mean field theory that cannot pretend to give the correct value of the critical temperature for all phase transitions. We pay attention that the relative change of $T_{c}$ under external pressure, the quantity $\frac{d \ln \left(T_{c}\right)}{d p}=\frac{1}{T_{c}} \frac{d T_{c}}{d p}$ is more reliable and may be compared with the experimental data. The doping dependence $\frac{1}{T_{c}(x)} \frac{d T_{c}(x)}{d p}$ obtained under the hydrostatic pressure $p=5.6 \mathrm{GPa}$ (such pressure corresponds to the relative decrease in the volume of $\epsilon=0.03$ ) is 
Table 2 The relative change in the area of the Fermi surfaces under pressure.

\begin{tabular}{llllll}
\hline \multirow{2}{*}{ type of pressure } & \multicolumn{5}{c}{ hole doping $x$} \\
\cline { 2 - 6 } & 0.145 & 0.15 & 0.151 & 0.16 & 0.22 \\
\hline hydrostatic pressure & $-7.076 ; 92.932(3)$ & $-14.07 ; 85.941(3)$ & $2.128 ;-93.055(4)$ & $2.554 ; 1.071(4)$ & $13.458 ; 2.08(4)$ \\
in-plane pressure & $-1.2(1)$ & $-14.032 ; 85.941(3)$ & $2.151 ; 97.096(4)$ & $2.554 ; 1.071(4)$ & $-57.582 ;-8.913(4)$ \\
pressure along $c$-axis & $-1.2(1)$ & $0.0(1)$ & $-17.075 ; 82.933(2)$ & $-1.994 ;-82.912(4)$ & $-19.788 ;-3.063(4)$ \\
\hline
\end{tabular}

The number in the round brackets indicates which of the four possible cases sold:

(1) both of the Fermi surfaces (with and without pressure) are not cleaved; in the cell there is a number $\delta=\left(S^{(\text {pressure })}-\right.$ $\left.S^{(\text {undeformed })}\right) / S^{(\text {undeformed })} \cdot 100 \%$

(2) the Fermi surface for undeformed case is cleaved and the Fermi surface for the case with the pressure is not cleaved; in the cell there are numbers $\delta_{\text {small(large })}=\left(S^{(\text {pressure })}-S_{\text {small(large })}^{\text {(undeform })} / S^{(\text {pressure })} \cdot 100 \%\right.$;

(3) the Fermi surface for undeformed case is not cleaved and the Fermi surface for the case with the pressure is cleaved; in the cell there are numbers $\delta_{\text {small (large })}=\left(S_{\text {small(large })}^{(\text {pressure }}-S^{(\text {undeformed })}\right) / S^{(\text {undeformed) }} \cdot 100 \%$;

(4) both of the Fermi surfaces (with and without pressure) are cleaved; in the cell there are numbers $\delta_{\text {small(large) }}=$ $\left(S_{\text {small(large) }}^{(\text {pressure }}-S_{\text {small(large) }}^{\text {(undeformed) }}\right) / S_{\text {small(large) }}^{(\text {undeformed) }} \cdot 100 \%$.

shown in Fig. 4a. It can be seen that the order of the quantity $\frac{1}{T_{c}} \frac{d T_{c}}{d p}$ coincide with the experimentally found value $\left(\frac{1}{T_{c}} \frac{d T_{c}}{d p}\right)_{\text {experiment }}=0.04 G P a^{-1}$ at $x=0.15[6]$.

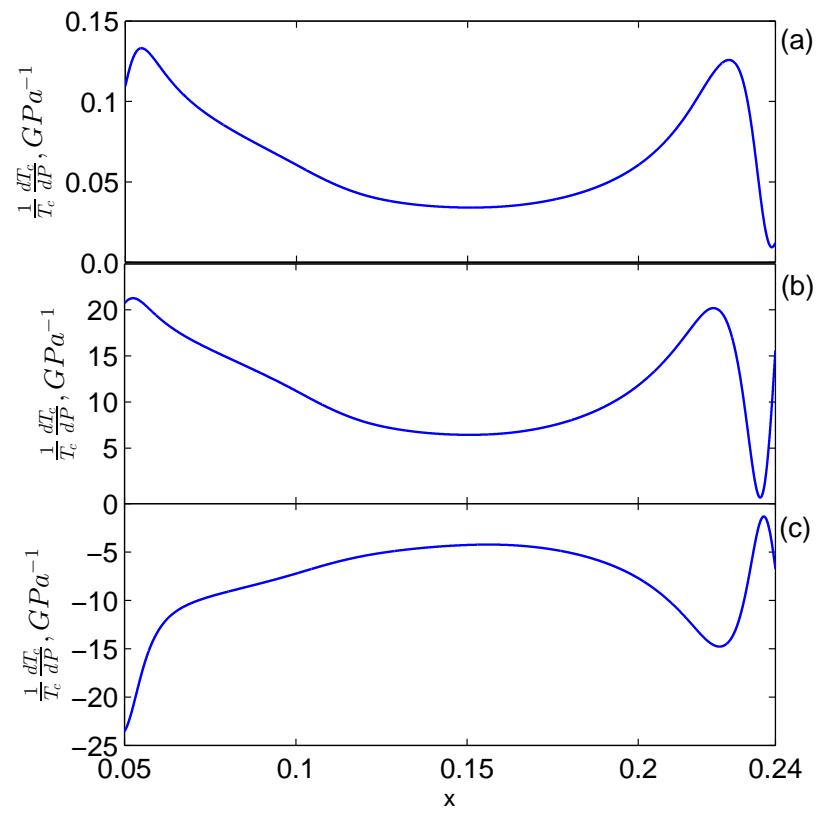

Figure 4 The relative change of the superconducting temperature $T_{c}$ under external pressure versus hole concentration $x$. The doping dependence $\frac{1}{T_{c}(x)} \frac{d T_{c}(x)}{d p}$ obtained under (a) the hydrostatic pressure $p=5.6 G P a$, (b) pressure along the $c$-axis and (c) pressure in the $a-b$ plane.

Let us now discuss the change of $T_{c}$ under pressure along the $c$-axis and in the $a$ - $b$ plane. Since we could not find in the literature the compressibility factors for these two types of pressure, we limited by the performance of the dependencies $\frac{1}{T_{c}(x)} \frac{d T_{c}(x)}{d \epsilon_{a-b}}$ and $\frac{1}{T_{c}(x)} \frac{d T_{c}(x)}{d \epsilon_{c}}$ (Fig. 4b and 4c) not bringing dependencies $\frac{1}{T_{c}(x)} \frac{d T_{c}(x)}{d p_{a-b}}$ and $\frac{1}{T_{c}(x)} \frac{d T_{c}(x)}{d p_{c}}$. We only mark that the signs of derivatives $\frac{1}{T_{c}(x)} \frac{d T_{c}(x)}{d p_{a-b}}$ and $\frac{1}{T_{c}(x)} \frac{d T_{c}(x)}{d p_{c}}$ are opposite to each other and are equal to the signs found in experimental studies [6].

7 Conclusions The experimental studies of high- $T_{c}$ cuprates properties under external pressure are mostly concerned with the critical temperature and structural parameters dependence on pressure. Here we have considered more wide range of properties: the electronic structure, the superexchange interaction and the critical temperature. At moderate pressure $\sim 10 \mathrm{GPa}$ the deformation of lattice is rather weak, just several percents. The changes of the electronic structure parameters are also quite small. We have calculated the effect of pressure on the area of the Fermi surface that can be verified by quantum oscillations measurements and predict strong increase of this effect for the doping close to the critical concentration $x_{c 1}=0.15$ and $x_{c 2}=0.24$ of the Lifshitz transition. The effect of pressure on the superexchange interaction is more stronger and has different signs for the deformations along $c$-axis and in $(a, b)$-plane. The same different signs we have obtained for the relative change of the $T_{c}$ under anisotropic deformations. For the isotropic pressure the relative change of $T_{c}$ is in a good agreement to the experimental data. The main contribution to the $T_{c}$ shift under external pressure results from the effect of pressure on the superexchange interaction that is the coupling in the magnetic mechanism of pairing. The electron-phonon interaction contribution to the $T_{c}$ is still under debates; certainly it also will be affected by the external pressure. Nevertheless we do not know now how to estimate it reliably, that is why this problem is outside of the scope of this paper. 
Acknowledgements We are thankful to Dr. M.M. Korshunov for useful discussions. This work was supported by RFBR Grants 13-02-01395, 14-02-00186, grant of Russian President NSh-2886.2014.2, and the Ministry of Education and Science of Russia (SibFU Government Contract for 2014-2016, No. 3085).

\section{References}

[1] J. S. Schilling, Handbook of High-Temperature Superconductivity: Theory and experiment (Springer, New York, 2007).

[2] A. K. Klehe, A. K. Gangopadhyay, J. Diederichs, and J. S. Schilling, Physica C 213, 266 (1993).

[3] L. Gao, Y. Y. Xue, F. Chen, Q. Xiong, R. L. Meng, D. Ramirez, C. W. Chu, J. H. Eggert, and H. K. Mao, Phys. Rev. B 50, 4260 (1994).

[4] F. Gugenberger, C. Meingast, G. Roth, K. Grube, V. Breit, T. Weber, H. Wuhl, S. Uchida, and Y. Nakamura, Phys. Rev. B 49, 13137 (1994).

[5] C. Meingast, A. Junod, and E. Walker, Physica C 272, 106 (1996).

[6] F. Hardy, N. J. Hillier, C. Meingast, D. Colson, Y. Li, N. Barisic, G. Yu, X. Zhao, M. Greven, and J. S. Schilling, Phys. Rev. Lett. 105, 167002 (2010).

[7] J. D. Jorgensen, D. G. Hinks, O. Chmaissem, D. N. Argyriou, J.F. Mitchell, and B. Dabrowski, Recent Developments in High Temperature Superconductivity (Springer, Berlin, 1996).

[8] H. Eisaki, N. Kaneko, D. L. Feng, A. Damascelli, P. K. Mang, K. M. Shen, Z. X. Shen, and M. Greven, Phys. Rev. B 69, 064512 (2004).

[9] Y. Ohta, T. Tohyama, and S. Maekawa, Phys. Rev. B 43, 2968 (1991).

[10] L. F. Feiner, J. H. Jefferson, and R. Raimondi, Phys. Rev. Lett. 76, 4939 (1996).

[11] E. Pavarini, I. Dasgupta, T. Saha-Dasgupta, O. Jepsen, and O. K. Andersen, Phys. Rev. Lett. 87, 047003 (2001).

[12] H. Sakakibara, H. Usui, K. Kuroki, R. Arita, and H. Aoki, Phys. Rev. Lett. 105, 057003 (2010).

[13] H. Sakakibara, K. Suzuki, H. Usui, K. Kuroki, R. Arita, D. J. Scalapino, and H. Aoki, Phys. Rev. B 86, 134520 (2012).

[14] X. J. Chen, V. V. Struzhkin, Z. Wu, R. J. Hemley, H. K. Mao, and H. Q. Lin, Phys. Rev. B 75, 134504 (2007).

[15] S.D. Conte, C. Gianetti, G. Coslovich, F. Cilento, D. Bossini, T. Abebaw, F. Banfi, G. Ferrini, H. Eisaki, M. Greven, A. Damascelli, D. van der Marel, and F. Parmigiani, Science 335, 1600 (2012).

[16] M. C. Aronson, S. B. Dierker, B. S. Dennis, S. W. Cheong, and Z. Fisk, Phys. Rev. B 44, 4657 (1991).

[17] M. I. Eremets, A. V. Lomsadze, V. V. Struzhkin, A. Maksimov, A. Puchkov, and I. I. Tartakovskii, JETP Lett. 54, 372 (1991).

[18] J. S. Schilling, Handbook of high temperature superconductivity: Theory and experiment (Springer Verlag, Hamburg, 2007).

[19] M. M. Korshunov, V. A. Gavrichkov, S. G. Ovchinnikov, I. A. Nekrasov, Z. V. Pchelkina, and V. I. Anisimov, Phys. Rev. B 72, 165104 (2005).
[20] S. G. Ovchinnikov, V. A. Gavrichkov, M. M. Korshunov, and E. I. Shneyder, LDA+GTB method for band structure calculations in the strongly correlated materials. In Theoretical Methods for strongly correlated systems. (Springer, Berlin, 2012).

[21] S. G. Ovchinnikov and I. S. Sandalov, Physica C 161, 607 (1989).

[22] V. A. Gavrichkov, S. G. Ovchinnikov, A. A. Borisov, and E. V. Goryachev, JETP 91, 369 (2000).

[23] V. A. Gavrichkov and S. G. Ovchinnikov, Physics of solid state 50, 1081 (2008).

[24] M. M. Korshunov and S. G. Ovchinnikov, Europ J. Physics B 57, 271 (2007).

[25] S. G. Ovchinnikov, E. I. Shneyder, and M. M. Korshunov, J. Phys.: Condens. Matter 23, 045701 (2011).

[26] Y. B. Gaididei and V. M. Loktev, Phys. Status Solidi B 147, 163 (1988).

[27] M. J. Akhtar, C. R. A. Catlow, S. M. Clark, and W. M. Temmerman, J. Phys. C: Solid State Phys. 21, 917 (1988).

[28] V. A. Gavrichkov and S. G. Ovchinnikov, Phys. Solidi State 40, 163 (1998).

[29] E. I. Shneyder, S. G. Ovchinnikov, M. M. Korshunov, and S. V. Nikolaev, JETP Letters 96, 349 (2012).

[30] H. Shimahara and S. Takada, J. Phys. Soc. Jpn 60, 2394 (1991).

[31] A. Barabanov and O. Starych, J. Phys. Soc. Jpn 61, 704 (1992).

[32] E. Dagotto, F.Ortolani, and D.Scalapino, Phys. Rev. B 46, 3183 (1992).

[33] Y. Ohta, K. Tsutsui, W. Koshibae, T. Shimozato, and S. Maekawa, Phys. Rev. B 46, 14022 (1992).

[34] W. Stephan and P. Horsch, Phys. Rev. Lett. 66, 2258 (1991).

[35] J. B. Grant and A. K. McMahan, Phys. Rev. Lett. 66, 488 (1991).

[36] S. G. Ovchinnikov, Physics Uspechi 40, 993 (1997).

[37] I. M. Lifshitz, M. Y. Asbel, and M. I. Kaganov, Electron Theory of Metals (Consultant Bureau, New York, 1973).

[38] N. Doiron-Leyraud, C.Proust, D. LeBoeuf, J. Levallois, J. B. Bonnemaison, R. Liang, D. A. Bonn, W. N. Hardy, and L. Taillefer, Nature 447, 565 (2007).

[39] S. Maekawa, T. Tohyama, S. E. Barnes, S. Ishibara, W. Koshibae, and G. Khaliullin, Physics of Transition Metal Oxides (Springer Series in Solid-State Sciences) (Springer, Hamburg, 2004).

[40] H. Eskes and J. H. Jefferson, Phys. Rev. B 48, 9788 (1993).

[41] R. Coldea, S. M. Hayden, G. Aeppli, T. G. Perring, C. D. Frost, T. E. Mason, S. W. Cheong, and Z. Fisk, Phys. Rev. Lett. 86, 5377 (2001).

[42] P. W. Anderson, Science 235, 1196 (1987).

[43] N. M. Plakida, V. Y. Yushankhai, and I. V. Stasyuk, Physica C 160, 80 (1989). 